\title{
Change in the Foveal Avascular Zone and Macular Capillary Network Density after Hyperbaric Oxygen Therapy in Healthy Retina
}

\author{
Sadık Görkem Çevik ${ }^{1}$, MD; Bekir Selim Bağıı², MD \\ ${ }^{1}$ Department of Ophthalmology, Yuksek Ihtisas Education and Training Hospital, Bursa, Turkey \\ ${ }^{2}$ Department of Underwater and Hyperbaric Medicine, Yuksek Ihtisas Education and Training Hospital, Bursa, Turkey \\ ORCID: \\ Sadık Görkem Çevik: https://orcid.org/0000-0002-5653-5800 \\ Bekir Selim Bağlı: https://orcid.org/0000-0002-0942-2619
}

\section{Abstract}

Purpose: This study aimed to evaluate responses in retinal tissue by swept source OCT angiography (OCT-A) to hyperoxia after hyperbaric oxygen ( $\mathrm{HBO} 2)$ therapy.

Methods: The study was conducted in volunteers who received $\mathrm{HBO} 2$ treatment but did not have any eye disease. Patients underwent detailed eye examinations including dilated fundus examination, visual acuity, and refraction before being admitted for $\mathrm{HBO} 2$ therapy. Measurements were made before and immediately after $\mathrm{HBO} 2$ therapy. Enface images of the retinal vasculature were obtained from the superficial and deep retinal plexus (SP/DP). Quantitative analysis of the vessel density (VD) and foveal avascular zone (FAZ) area was performed.

Results: In total, 31 patients (15 female) with healthy retina were included in the study. The mean age was 42.8 years. The mean SP vascular density measurements before $\mathrm{HBO} 2$ therapy for the right and left eyes were $15.18 \pm 1.2$ $\mathrm{mm}^{-1}$ and $15.01 \pm 1.3 \mathrm{~mm}^{-1}$, respectively; the measurements after HBO2 therapy for the right and left eyes were $14.34 \pm 1.4 \mathrm{~mm}^{-1}$ and $14.48 \pm 1.19 \mathrm{~mm}^{-1}$. The mean DP vascular density measurements before $\mathrm{HBO} 2$ therapy for the right and left eyes were $16.03 \pm 1.69 \mathrm{~mm}^{-1}$ and $16.1 \pm 1.45 \mathrm{~mm}^{-1}$, respectively; the measurements after $\mathrm{HBO} 2$ therapy for the right and left eyes were $15.02 \pm 1.65$ $\mathrm{mm}^{-1}$ and $15.12 \pm 2.16 \mathrm{~mm}^{-1}$, respectively. Reduction of mean VD in superficial and deep plexus after $\mathrm{HBO} 2$ was statistically significant $(P=0.001$ and $P=0.000$, respectively). Changes in mean $\mathrm{FAZ}$ area before and after $\mathrm{HBO} 2$ therapy were not statistically significant $(P=0.719)$.

Conclusion: The healthy retina responds to oxygen supersaturation with $\mathrm{HBO} 2$ therapy by eventually decreasing vascular density in all layers. These findings may be important for further studies especially related to retina and choroidal oxygenation.

Keywords: Hyperbaric Oxygen; OCT-A; Retinal Ischemic Disease 


\section{INTRODUCTION}

Hyperbaric oxygen (HBO2) therapy is a medical treatment method that aims to increase the level of dissolved oxygen in tissues. Patients intermittently breathe near 100\% oxygen while inside a hyperbaric chamber that is pressurized to greater than sea level pressure (1 atmosphere absolute [ATA]) and high tissue oxygen level remains high for up to $4 \mathrm{hr}$ after the therapy. ${ }^{[1]}$ Retina blood flow is provided by two primary sources: the retinal artery and the choroid. The retina has an internal autoregulatory system that preserves blood flow and stable oxygenation by altering it in response to unexpected conditions. ${ }^{[2]}$ This regulatory system changes blood flow and vascular density for unexpected conditions and may not work correctly under some conditions, such as diabetic retinopathy or age-related macular degeneration. ${ }^{[3-9]}$

Macular microvasculature is a complex system consisting of three capillary plexuses for the blood supply of the inner retina: the superficial retinal plexus (SP) located in the retinal nerve fiber layer and the two-plexus located at the inner and outer border of the inner nuclear layer (INL), which constitute the deep retinal plexus (DP). ${ }^{[10]}$ Noninvasive retinal blood-flow imaging techniques, such as scanning laser Doppler and Doppler OCT, have previously been described..$^{[1-14]}$ OCT angiography (OCT-A) is a novel, noninvasive technique for retinal vasculature imaging that allows segmentation and quantification of the retinal microvasculature. ${ }^{[15]}$

The present study aimed to evaluate changes in foveal avascular zone (FAZ) area and perifoveal capillary network density in SP and DP, with the aid of swept source OCT-A in patients undergoing HBO2 therapy.

Correspondence to:

Sadık Görkem Çevik, MD. Yüksek ihtisas Education and Training Hospital, Bursa 16310, Turkey.

E-mail: gorkemcevik@hotmail.com

Received: 27-11-2020Ａccepted: 17-02-2021

Access this article online

Website: https://knepublishing.com/index.php/JOVR

DOI: 10.18502/jovr.v16i3.9436

\section{METHODS}

This prospective, interventional study was handled between April 2017 and October 2017 after the ethics committee of hospital approved the protocol. The study adhered to the Declaration of Helsinki, and patients were informed about the treatment options and possible complications. The preprocedural written informed consent was obtained from patients. The study was carried on in volunteers who did not have any known eye disease and who received $\mathrm{HBO} 2$ treatment for other reasons. Volunteer patients underwent detailed eye examinations that included dilated fundus examination and visual acuity and refraction before they were admitted for HBO2 therapy. Exclusion criteria were: age $<18$ years; any ocular diseases; inability to maintain stable fixation for scanning; visual acuity worse than 20/40; refractive errors $>-4.00$ or +2.00 diopters; media opacity; history of ocular surgery; and systemic diseases that may affect microcOirculation (e.g., diabetes mellitus, hypertension).

Measurements were made using the DRI OCT Triton (Topcon Co- Tokyo-Japan) system before and immediately (approximately $1 \mathrm{~min}$ ) after the fifth session of HBO2 therapy (every day one session). FAZ, superficial vascular plexus (SP) [Figure 1] and deep vascular [Figure 3] plexus (DP) density measurements were made.

The DRI OCT Triton system uses a swept-source laser with a center wavelength of $1050 \mathrm{~nm}$ and a scan speed of 100,000 A-scans per second. OCT-A is based on the Topcon OCT Angiography ratio analysis (OCTARA) algorithm. This system distinguishes mobile blood flow from tissue using motion contrast measure. An active eye tracker was used to decrease motion artifacts during OCTA imaging. Each $3 \times 3 \mathrm{~mm}$ volume scan contained $320 \times 320$ pixels. In the present study, we applied automated layer segmentation to SP (from $2.6 \mu \mathrm{m}$ below the internal limiting membrane to $15.6 \mu \mathrm{m}$ below the junction between the inner plexiform and inner nuclear layers [IPL/INL]) and DP (from

This is an open access journal, and articles are distributed under the terms of the Creative Commons Attribution-NonCommercial-ShareAlike 4.0 License, which allows others to remix, tweak, and build upon the work non-commercially, as long as appropriate credit is given and the new creations are licensed under the identical terms.

How to cite this article: Cevik SG, Bağlı BS. Change in the Foveal Avascular Zone and Macular Capillary Network Density after Hyperbaric Oxygen Therapy in Healthy Retina. J Ophthalmic Vis Res 2021;16:393-399. 


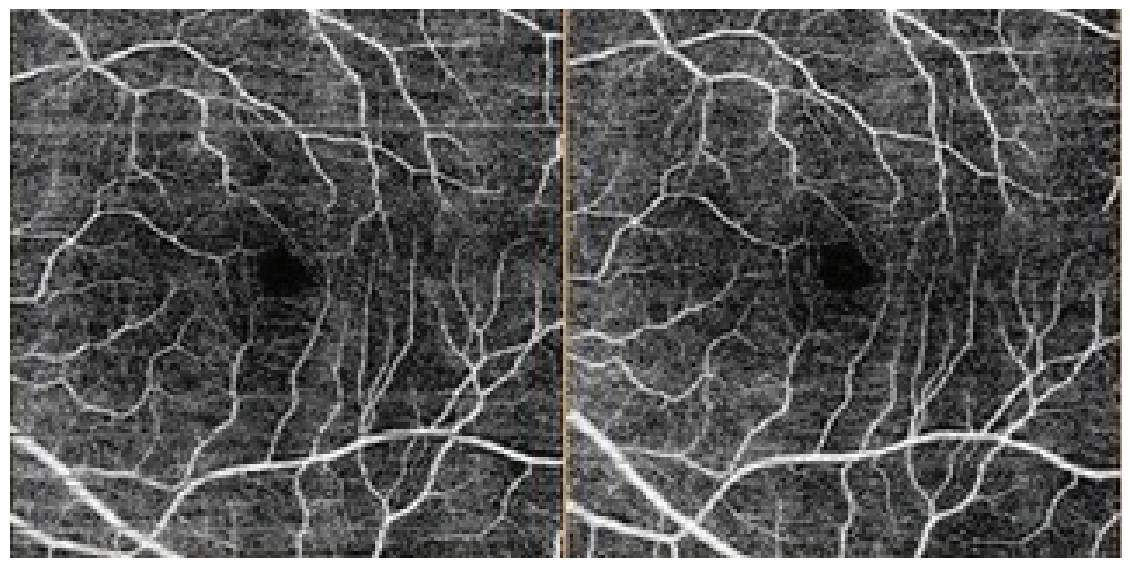

Figure 1. Swept source OCT-A image before (left) and after (right) HBO2 treatment (superficial plexus).

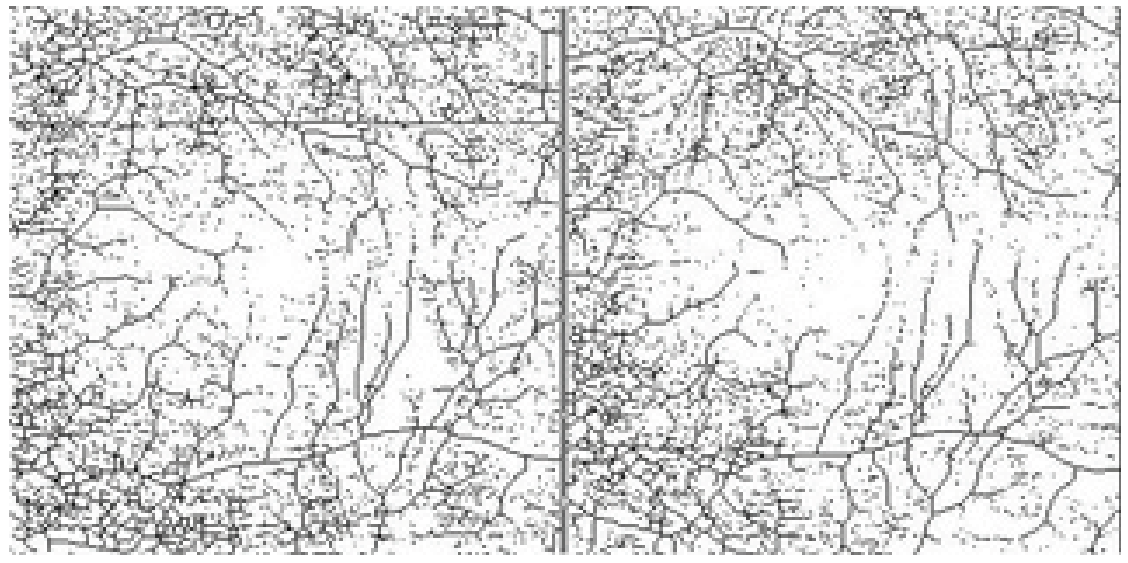

Figure 2. Skeletonized vessel maps of the superficial plexus image before (left) and after (right) HBO2 treatment.

15.6 $\mu \mathrm{m}$ below of IPL/INL to $70.2 \mu \mathrm{m}$ below the IPL/INL). All measurements were obtained between 9:00 AM and 12:00 AM to avoid the effects of diurnal variations and blood pressure and pulse were recorded. Poor-quality OCT-A due to blinking or fixation loss were excluded from the study. The qualitative examination of the OCT-A of the SP and DP were then independently performed by two masked examiners (Dr Akdogan and Dr Tok).

Quantitative analysis of $3 \times 3 \mathrm{~mm}$ OCT angiograms, including FAZ area and capillary vessel density (CVD; i.e., SP-DP) measurement, was performed using ImageJ software (developed by Wayne Rasband, National Institutes of Health, Bethesda, MD; available at http://rsb.info.nih.gov/ij/index.html). The original OCTA images of the SP and the DP were binarized to convert them from grayscale into black and white images using the ImageJ software, and the FAZ area was manually marked and measured in original images of SP.
To measure the CVD, the binarized OCTA images were skeletonized, showing the blood vessels as a 1-pixel-wide line, and ImageJ was used to count the number of black pixels and total pixels [Figures 2 and 4]. CVD was then calculated as ([pixels of vessels,3/256])/(area in squared millimeter) in $\mathrm{mm}^{-1}$ and this method was described by Khairallah et al previously. ${ }^{[27]}$ Cases with projection artifact were excluded from the analysis of vessel density. HBO2 therapy was administered in multiple chambers at 2.4 ATA. Each session of $\mathrm{HBO} 2$ therapy lasted about $120 \mathrm{~min}$ composed of three 25-min oxygen periods during which patients breathed $100 \%$ oxygen at 2.4 ATA, separated by 5 -min air brakes, with compression and decompression during the remaining time.

Data analysis was performed using SPSS Statistics for Windows, version 22.0 (SPSS Inc., Chicago, IL, United States). All differences associated with a chance probability of 0.05 or less were considered statistically significant. Statistical 


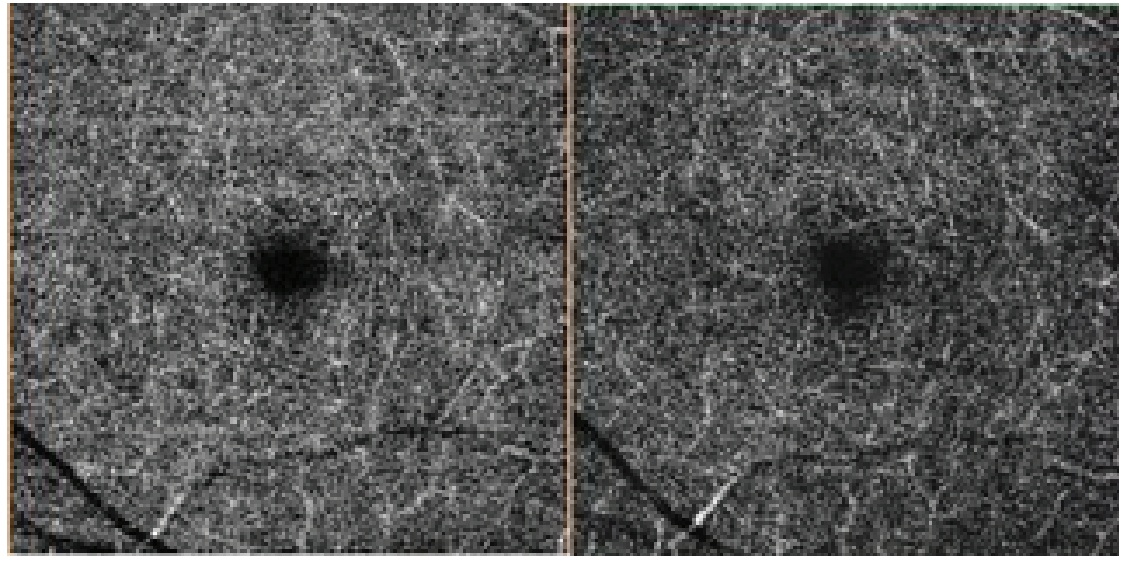

Figure 3. Swept source OCT-A image before (left) and after (right) HBO2 treatment (deep plexus).

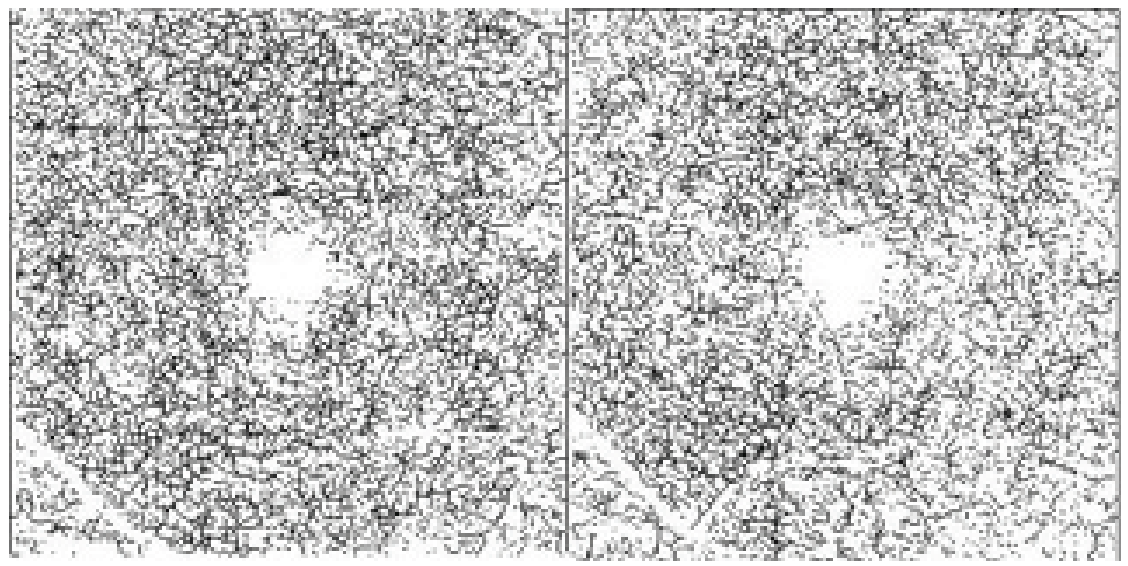

Figure 4. Skeletonized vessel maps of the deep plexus image before (left) and after (right) HBO2 treatment.

significance between before and after $\mathrm{HBO} 2$ therapy was tested with dependent samples $t$-test.

\section{RESULTS}

This study included 31 patients (15 female, 48\%) with healthy retinas. The mean age was $42.8 \pm 15.3$ (19-73) years. Indications of HBO2 therapy were femur bone avascular necrosis (15 patients, 48\%), chronic osteomyelitis (8 patients, $25 \%$ ), idiopathic sudden sensorineural hearing loss (6 patients, $19 \%)$, malign otitis externa (1 patient, 3\%), crush injury (1 patient, 3\%), as summarized in Table 1.

The mean SP vascular density measurements before HBO2 therapy for the right and left eyes were $15.18 \pm 1.2 \mathrm{~mm}^{-1}\left(\min 13.1 \mathrm{~mm}^{-1}-\max 17.1\right.$ $\left.\mathrm{mm}^{-1}\right)$ and $15.01 \pm 1.3 \mathrm{~mm}^{-1}\left(\min 13.3 \mathrm{~mm}^{-1}\right.$ $\max 17.2 \mathrm{~mm}^{-1}$ ) respectively; the measurements after $\mathrm{HBO} 2$ therapy for the right and left eyes were $14.34 \pm 1.4 \mathrm{~mm}^{-1}\left(\min 12.1 \mathrm{~mm}^{-1}-\max 16.2\right.$ $\left.\mathrm{mm}^{-1}\right)$ and $14.48 \pm 1.19 \mathrm{~mm}^{-1},\left(\min 12.3 \mathrm{~mm}^{-1}-\right.$ max $16.2 \mathrm{~mm}^{-1}$ ) respectively. These changes were statistically significant $(P=0.001$ and $P=0.001$, respectively).

The mean DP vascular density measurements before $\mathrm{HBO} 2$ therapy for the right and left eyes were $16.03 \pm 1.69 \mathrm{~mm}^{-1}\left(\min 13.82 \mathrm{~mm}^{-1}-\max \right.$ $\left.18.12 \mathrm{~mm}^{-1}\right)$ and $16.1 \pm 1.45 \mathrm{~mm}^{-1}\left(\min 13.82 \mathrm{~mm}^{-1}\right.$ max $18.03 \mathrm{~mm}^{-1}$ ), respectively; the measurements after $\mathrm{HBO} 2$ therapy for the right and left eyes were $15.02 \pm 1.65 \mathrm{~mm}^{-1}\left(\min 13.23 \mathrm{~mm}^{-1}-\max \right.$ $\left.17.05 \mathrm{~mm}^{-1}\right)$ and $15.12 \pm 2.16 \mathrm{~mm}^{-1}(\min 12.10$ $\mathrm{mm}^{-1}-\max 18.23 \mathrm{~mm}^{-1}$ ), respectively. These changes were statistically significant $(P<0.000$ and $P<0.000$, respectively). (Measurement data before and after HBO2 therapy are shown in Table 2.)

The mean FAZ area for the right eye was 0.363 $\pm 0.15(0.20-0.62) \mathrm{mm}^{2}$ before HBO2 therapy and $0.372 \pm 0.34(0.21-0.49) \mathrm{mm}^{2}$ after the therapy. The mean FAZ area for the left eye was $0.354 \pm 0.32$ $(0.28-0.47) \mathrm{mm}^{2}$ before HBO2 therapy and 0.357 
Table 1. Indications of $\mathrm{HBO} 2$ therapy for patients

Indications

Number of patients

Femur bone avascular necrosis

15 (48\%)

Chronic osteomyelitis

8 (25\%)

Idiopathic sudden sensorineural hearing loss

$6(19 \%)$

Severe otitis externa

1 (3\%)

Crush injury

1 (3\%)

Total

31

Table 2. Measurement data before and after $\mathrm{HBO} 2$ therapy

\begin{tabular}{|c|c|c|c|}
\hline & Before $\mathrm{HBO} 2$ therapy & After HBO2 therapy & $P$-value \\
\hline Mean right eye superficial capillary density $\left(\mathrm{mm}^{-1}\right)$ & $15.18 \pm 1.2$ & $14.34 \pm 1.4$ & 0.001 \\
\hline Mean right eye deep capillary density $\left(\mathrm{mm}^{-1}\right)$ & $16.03 \pm 1.69$ & $15.02 \pm 1.65$ & 0.000 \\
\hline Mean left eye superficial capillary density $\left(\mathrm{mm}^{-1}\right)$ & $15.01 \pm 1.3$ & $14.48 \pm 1.19$ & 0.001 \\
\hline Mean left eye deep capillary density $\left(\mathrm{mm}^{-1}\right)$ & $16.1 \pm 1.45$ & $15.12 \pm 2.16$ & 0.000 \\
\hline Mean right eye $F A Z\left(\mathrm{~mm}^{2}\right)$ & 0.363 & 0.372 & 0.374 \\
\hline Mean left eye FAZ $\left(\mathrm{mm}^{2}\right)$ & 0.354 & 0.357 & 0.719 \\
\hline
\end{tabular}

$\mathrm{mm}^{2} \pm 0.22(0.23-0.46)$ after the therapy. These changes were not statistically significant $(P=0.374$, $P=0.719$ ).

\section{DISCUSSION}

In the present study, we aimed to investigate the effect of five sessions $\mathrm{HBO} 2$ therapy on the retina. We found significant decreases in SP and DP capillary network density in the macular region. However, there was no effect on FAZ measurements.

The retina has unique dual circulation and complicated autoregulatory systems. The outer retina and FAZ are nourished with diffusion from the choroidal circulation (CC). ${ }^{[20,26]}$ Responses to hypoxia in retinal artery circulation (RC) and $C C$ are different. ${ }^{[18]} \mathrm{Yi}$ et al reported that $\mathrm{CC}$ responds poorly in hypoxia conditions. ${ }^{[19]}$

Linsenmeier and Braun found that the inner retina was well-preserved under hypoxic condition by the help of increased RC during hypoxia, while blood flow in CC remained unchanged in cats' retina. ${ }^{[20]}$ Retinal response to hyperoxia is different between mammalian species. The rat, rabbit, and guinea pig that are commonly demanded mammalian in ophthalmic research and they have different responses to systemic hyperoxia. ${ }^{[3]}$
Hyperoxia changes total human retinal blood flow, as measured by other measurement methods before. ${ }^{[13-16]}$ The autoregulatory response protects retinal tissue from the adverse effects of hypoxia and hyperoxia. Hagag et al found that hyperoxia caused a significant decrease in the flow index $(11 \%)$ and the VD of the deep capillary plexus (DP) of the retina (7.8\%). However, decreases in the vessel densities of the superficial and intermediate plexuses were not statistically significant. In Hagag et al's study, oxygen was supplied with a face mask for individuals; the mask flow rate was $15 \mathrm{l} / \mathrm{min}{ }^{[17]}$ Hagag et al hypothesized that the response of DP in different oxygen concentrations could be significant and essential because CC did not respond like DP and the blood flow of the SP also decreased in over-oxygenation but supplying oxygen with a face mask may not be enough to produce a statistically significant decrease. Contrary to Hagag et al's study, reduction in both SP and DP VD was statistically significant in our study. We think that this may be the result of a high amount of dissolved oxygen in the plasma that was supplied by $\mathrm{HBO} 2$. $\mathrm{HBO} 2$ therapy increased dissolved oxygen levels in all retinal layers, including the superficial retinal layers. During $\mathrm{HBO} 2$, the oxygen concentration in the avascular outer retina increases due to higher 
supply from the CC, which maintains constant flow at high $\mathrm{O}_{2}$ level. To preserve steady oxygen flux in the outer retina, the DP must compensate with greater vasoconstriction. ${ }^{[25,28-30]}$

In the present study, the changes in FAZ before and after $\mathrm{HBO} 2$ therapy were not statistically significant. FAZ area changes can be seen in retinal disease and five $\mathrm{HBO} 2$ sessions may be short to describe certain effect of HBO2 on FAZ. ${ }^{[21-24]}$

The limitations of our study are: small sample size, limited number of $\mathrm{HBO} 2$ sessions, measurement of vascular plexus density by an external software, and assessment of only $3 \times 3$ $\mathrm{mm}$ macular area.

In summary, swept source OCT-A evaluate retinal vascular plexus and FAZ and we reported a significant decrease of foveal capillary network density in SP and DP with $\mathrm{HBO} 2$ therapy. This finding gives us new evidence regarding the interaction between choroidal and retinal circulation and offers us new perspective about retinal oxygenation with $\mathrm{HBO} 2$ treatment.

\section{Acknowledgement}

The authors are thankful to Dr Mediha Tok and Dr Muberra Akdogan for evaluating images.

\section{Financial Support and Sponsorship}

Nil.

\section{Conflicts of Interest}

There is no conflict of interest.

\section{REFERENCES}

1. Weaver LK. Hyperbaric oxygen therapy indications. 13th ed. Durham, NC: Best Publishing, 2014. p. 1-8, 247.

2. Rassam SM, Patel V, Chen HC, Kohner EM. Regional retinal blood flow and vascular autoregulation. Eye 1996;10:331337.

3. Cringle SJ, Yu DY. Regulation of oxygen tension in the mammalian retina during systemic hyperoxia is species dependent. Adv Exp Med Biol 2018;1072:241-244.

4. Friedman E. A hemodynamic model of the pathogenesis of age-related macular degeneration. Am J Ophthalmol 1997;124:677-682.

5. Schmetterer L, Wolzt M. Ocular blood flow and associated functional deviations in diabetic retinopathy. Diabetologia 1999;42:387-405.
6. Kohner EM, Patel V, Rassam SM. Role of blood flow and impaired autoregulation in the pathogenesis of diabetic retinopathy. Diabetes 1995;44:603-607.

7. Pemp B, Schmetterer L. Ocular blood flow in diabetes and age-related macular degeneration. Can J Ophthalmol 2008;43:295-301.

8. Gilmore ED, Hudson C, Nrusimhadevara RK, Harvey PT, Mandelcorn M, Lam WC, et al. Retinal arteriolar diameter, blood velocity, and blood flow response to an isocapnic hyperoxic provocation in early sight-threatening diabetic retinopathy. Invest Ophthalmol Vis Sci 2007;48:17441750.

9. Remsch H, Spraul CW, Lang GK, Lang GE. Changes of retinal capillary blood flow in age-related maculopathy. Graefes Arch Clin Exp Ophthalmol 2000;238:960-964.

10. Strenn K, Menapace R, Rainer G, Findl O, Woltz M. Reproducibility and sensitivity of scanning laser doppler flowmetry during graded changes in PO2. Br J Ophthalmol 1997;81:360-364.

11. Riva CE, Grunwald JE, Sinclair SH. Laser Doppler velocimetry study of the effect of pure oxygen breathing on retinal blood flow. Invest Ophthalmol Vis Sci 1983;24:47-51.

12. Bower BA, Zhao M, Zawadzki RJ, Izatt JA. Real-time spectral domain Doppler optical coherence tomography and investigation of human retinal vessel autoregulation. J Biomed Opt 2007;12:041214.

13. Pechauer AD, Huang $D$, Jia $Y$. Detecting blood flow response to stimulation of the human eye. BioMed Res IntI 2015;2015:121973.

14. Pechauer AD, Tan O, Liu L, Jia Y, Hou V, Hills W, et al. Retinal blood flow response to hyperoxia measured with en face doppler optical coherence tomography. Invest Ophthalmol Vis Sci 2016;57:OCT141-OCT145.

15. Jia Y, Tan O, Tokayer J, Potsaid B, Wang Y, Liu JJ, et al. Split-spectrum amplitude decorrelation angiography with optical coherence tomography. Opt Express 2012;20:4710-4725.

16. Tam J, Dhamdhere KP, Tiruveedhula P, Manzanera S, Barez S, Bearse MA Jr, et al. Disruption of the retinal parafoveal capillary network in type 2 diabetes before the onset of diabetic retinopathy. Invest Ophthalmol Vis Sci 2011;52:9257-9266.

17. Hagag, AM, Pechauer AD, Liu L, Wang J, Zhang M, Jia M, et al. OCT angiography changes in the 3 parafoveal retinal plexuses in response to hyperoxia. Ophthalmol Retina 2018;2:329-336.

18. Lange CA, Bainbridge JW. Oxygen sensing in retinal health and disease. Ophthalmologica 2012;227:115-131.

19. Yi J, Liu W, Chen S, Backman S, Shebani N, Sorenson $\mathrm{CM}$, et al. Visible light optical coherence tomography measures retinal oxygen metabolic response to systemic oxygenation. Light Sci App/ 2015;4:e334.

20. Linsenmeier RA, Braun RD. Oxygen distribution and consumption in the cat retina during normoxia and hypoxemia. J Gen Physiol 1992;99:177-197.

21. Nelson DA, Burgansky-Eliash Z, Barash $\mathrm{H}$, Loewenstein A, Barak A, Bartov $E$, et al. High-resolution wide-field imaging of perfused capillaries without the use of contrast agent. Clin Ophthalmol 2011;5:1095-1106. 
22. Kim DY, Fingler J, Zawadzki RJ, Park SS, Morse LS, Schwardz DM, et al. Noninvasive imaging of the foveal avascular zone with high-speed, phase-variance optical coherence tomography. Invest Ophthalmol Vis Sci 2012;53:85-92.

23. Tam J, Dhamdhere KP, Tiruveedhula P, Lujan BJ, Johnson $\mathrm{RN}$, Bearse MA Jr, et al. Subclinical capillary changes in nonproliferative diabetic retinopathy. Optom Vis Sci 2012;89:E692-E703.

24. Miyamoto K, Khosrof S, Bursell SE, Rohan R, Murata T, Clermont AC, et al. Prevention of leukostasis and vascular leakage in streptozotocin-induced diabetic retinopathy via intercellular adhesion molecule-1 inhibition. Proc Natl AcadSci USA 1999;96:10836-10841.

25. Wangsa-Wirawan ND, Linsenmeier RA. Retinal oxygen: fundamental and clinical aspects. Arch Ophthalmol 2003;121:547-557.

26. Snodderly DM, Weinhaus RS. Retinal vasculature of the fovea of the squirrel monkey Saimiri sciureus: three-dimensional architecture, visual screening, and relationships to the neuronal layers. J Comp Neurol 1990;297:145-163.

27. Khairallah M, Abroug N, Khochtali S, Mahmoud A, Jelliti B, Coscas G, et al. Optical coherence tomography angiography in patients with Behçet uveitis. Retina 2017;37:1678-1691.

28. Delaey C, van De Voorde J. Regulatory mechanisms in the retinal and choroidal circulation. Ophthalmic Res 2000;32:249-256.

29. Geiser MH, Riva CE, Dorner GT, Diermann U, Luksch A, Schmetterer L. Response of choroidal blood flow in the foveal region to hyperoxia and hyperoxia-hypercapnia. Curr Eye Res 2000;21:669-676.

30. Cringle SJ, Yu DY. A multi-layer model of retinal oxygen supply and consumption helps explain the muted rise in inner retinal $\mathrm{PO}(2)$ during systemic hyperoxia. Comp Biochem Physiol A Mol Integr Physiol 2002;132:61-66. 